\title{
A Catalog of OB Associations in the spiral galaxy NGC 300^
}

\author{
G. Pietrzyński ${ }^{1,5}$, W. Gieren ${ }^{1}$, P. Fouqué ${ }^{2,3}$, and F. Pont ${ }^{4}$ \\ 1 Universidad de Concepción, Departamento de Fisica, Casilla 160-C, Concepción, Chile \\ e-mail: wgieren@coma.cfm.udec.cl \\ 2 Observatoire de Paris-Meudon DESPA, 92195 Meudon Cedex, France \\ 3 European Southern Observatory, Casilla 19001, Santiago 19, Chile \\ e-mail: pfouque@eso.org \\ 4 Universidad de Chile, Departamento de Astronomia, Casilla 36D, Santiago, Chile \\ e-mail: FredericPont@obs.unige.ch \\ 5 Warsaw University Observatory, Al. Ujazdowskie 4, 00-478 Warszawa, Poland \\ Received 2 January 2001 / Accepted 13 March 2001
}

\begin{abstract}
We present results of a search for OB associations in NGC 300. Using an automatic and objective method (PLC technique) 117 objects were found. Statistical tests indicate that our sample is contaminated by less than 10 detections due to random concentrations of blue stars. Spatial distributions of detected associations and H II regions are strongly correlated. The size distribution reveals a significant peak at about $60 \mu \mathrm{rad}$ which corresponds to 125 parsecs if a distance modulus of $26.66 \mathrm{mag}$ is assumed. Besides the objects with sizes corresponding to typical associations we also found several much larger objects. A second level application of our detection method revealed that most of these are composed of smaller subgroups, with sizes of about $100 \mathrm{pc}$.
\end{abstract}

Key words. galaxies: NGC 300: star clusters - galaxies: stellar content - stars: early type

\section{Introduction}

OB associations constitute physical groups of young, massive, gravitationally unbound stars, which have formed in the same molecular cloud. Observations of these short lived objects provide much valuable information about regions of recent and/or current star formation, parent galaxies and star formation processes in general. In particular, a comparison of the properties of systems of associations in different galaxies may help us to better understand the influence of the environment on mechanisms of star formation.

A lot of work has been dedicated in the past to the detection and analysis of properties of associations in nearby galaxies. However, as thoroughly discussed by Hodge (1986), the identification of OB associations is a very difficult task. Indeed, due to very low surface density of stars it is not possible to detect them just based on the density contrast with the surrounding field. For a long time, associations were identified from visual searches of photographic plates. Subjective identification together with nonuniform observational material and poor

Send offprint requests to: G. Pietrzyński, e-mail: pietrzyn@hubble.cfm.udec.cl

* Based in part on observations obtained with the $1.3 \mathrm{~m}$ Warsaw telescope at the Las Campanas Observatory of the Carnegie Institution of Washington, and on observations obtained at the European Southern Observatory, La Silla, Chile. resolution often led to disagreement between different authors. As a result of these limitations it was not possible to perform unambiguous comparisons of the properties of stellar associations found in different galaxies. To overcome these difficulties new, automatic and objective techniques of detecting OB associations were developed (Battinelli 1991; Wilson 1991). All one needs in order to use these methods are the positions of the sample of OB stars. A successful application of these techniques to search for $\mathrm{OB}$ associations in several nearby galaxies (Battinelli 1991; Magnier et al. 1993; Wilson 1992) reflects that they are very useful tools for building uniform catalogues of $\mathrm{OB}$ associations in galaxies well suited for comparison purposes.

In this contribution we present the results of a search for $\mathrm{OB}$ associations in the nearly face-on spiral galaxy NGC 300. This galaxy, which is a member of the Sculptor Group, presents massive recent star formation, yet no systematic work to discover OB associations in this stellar system has been undertaken. To our knowledge only five stellar groups assumed to be associations were observed by Breysacher et al. (1997) during their search for WR stars in stellar associations. A systematic search with the aim to uncover the complete system of OB associations in this important nearby galaxy is therefore long overdue.

Our paper is organized as follows. In Sect. 2 we briefly describe the observations and data reduction techniques 
applied in this work. The algorithm used for searching for potential associations is described in Sect. 3. Results are presented in Sect. 4. Section 5 contains some final remarks on our findings.

\section{Observations}

The observational data have been collected in the course of a long term project whose principal goal is to discover a large number of Cepheid variables in NGC 300 in order to calibrate the effect of metallicity on Cepheid luminosities. Up to now several hundred images in $B V R I$ bands were collected with the Wide Field Camera attached to the ESO/MPI $2.2 \mathrm{~m}$ telescope on La Silla. This camera has a pixel scale of $0.238 \mathrm{arcsec} / \mathrm{pixel}$, and its large field of view of $33 \times 34$ arcmin allows to cover the whole galaxy with one pointing. For the search of $\mathrm{OB}$ associations we used $10 \mathrm{~B}$ and $10 \mathrm{~V}$ images obtained on two photometric nights, at low airmass and under 0.8 arcsec seeing conditions. Exposure time was set to $360 \mathrm{~s}$ for all images in each filter. As can be seen from Fig. 1 the limiting magnitude was about $22.5 \mathrm{mag}$ in $B$ and about $23 \mathrm{mag}$ in $V$. After debiasing and flatfielding the images with the IRAF $^{1}$ package, each of the eight $2 \mathrm{k} \times 4 \mathrm{k}$ chips was divided into 16 slightly overlapping sub-frames. Profile photometry with DAOPHOT and ALLSTAR programs was performed for all stars detected in all subframes. Then the photometry on all subframes was tied together using stars located in the overlapping regions.

In order to calibrate our data we performed follow-up observations with the Warsaw $1.3 \mathrm{~m}$, photometric telescope located at Las Campanas Observatory, Chile. The telescope was equipped with a $2048 \times 2048$ CCD camera. The scale is $0.41 \mathrm{arcsec} / \mathrm{pixel}$, which corresponds to a field of view of about $14.5 \times 14.5$ arcmin. More details about the instrumental system can be found in Udalski et al. (1997). Observations were conducted through $B V R I$ filters, during 2 photometric nights. We monitored four fields, covering about $25 \times 25$ arcmin of the central part of NGC 300. During each night a large number of Landolt standard stars (Landolt 1992) covering a wide range of colors and airmasses, were also observed in order to tie our new secondary standards in NGC 300 to the Landolt system. Preliminary reductions (i.e. debiasing and flatfielding) were performed with the IRAF package while the PSF photometry was done with the DAOPHOT/ALLSTAR package.

The accuracy of the transformation of the instrumental magnitudes and colors to the standard system is about 0.04 mag for the $B$ and $V$ bands, and slightly better for the $(B-V)$ color. As a next step we carefully selected objects with reliable photometry and used them to calibrate the photometry from the WFI camera. In this process,

\footnotetext{
${ }^{1}$ IRAF is distributed by the National Optical Astronomy Observatories, which are operated by the Association of Universities for Research in Astronomy, Inc., under cooperative agreement with the NSF.
}

we eliminated background galaxies, severely blended and saturated stars by visual examination.

Having established a sequence of about 300 photometric secondary standard stars in the field of NGC 300 (results to be published in a forthcoming paper), we transformed the WFI photometry from each chip using a least squares method. Usually more than 20 stars common between a given chip and our catalog of secondary standards were identified, and the accuracy of internal transformations was better than $0.01 \mathrm{mag}$ (rms).

We are continuing the observations of secondary standards stars in NGC 300, and the accuracy of their brightness calibration will soon be significantly improved. A more detailed description of the project, observations, and reduction procedures will be presented in a followup paper.

\section{Search for associations}

\subsection{Identification method}

The Path Linkage Criterion (PLC) technique (Battinelli 1991) was adopted in our search for OB associations in NGC 300. For a detailed description of this technique the reader is referred to Battinelli (1991). In short, this method assumes that any given two stars in an ensemble of OB stars belong to the same association if and only if it is possible to connect these two stars, by successively linking OB stars located between them, separated from each other by no more than a certain fixed distance parameter, or search radius, called $d_{\mathrm{s}}$. The advantage of the PLC method is that $d_{\mathrm{s}}$ can be unambiguously derived based on the given catalog of early type stars using the function $f_{p}(d)$. This function describes the number of groups containing at least $p$ stars, for any given value of the distance parameter $d$. The behaviour of this function is as follows: a steep rise until a maximum value is reached, followed by a gradual decrease towards a limiting value of 1 , which corresponds to the situation that the value of $d$ is so large that all stars can be connected using it (i.e. all stars are assigned to just one group). The optimum value of the distance parameter $d_{\mathrm{s}}$ is defined as the value of $d$ corresponding to the maximum of the function $f_{p}(d)$.

\subsection{Application of the PLC technique to NGC 300}

Before we can start to search for OB associations we need to select the catalog of blue stars. In the case of the photometric data it means that we need to adopt brightness and color cutoffs. The sample of blue stars was selected from the photometric catalog of stars in NGC 300 using the following criteria: $V<22 \mathrm{mag}$ and $-0.6<B-V<$ $0.4 \mathrm{mag}$. We note that NGC 300 has a very low foreground reddening, in the order of $E(B-V)=0.02 \mathrm{mag}$, so the color cutoff for the unreddened $B-V$ color is also approximately 0.4 mag. Altogether 4016 stars satisfied our selection criteria and entered our list of blue stars. Unfortunately the distance to NGC 300 is rather uncertain 
at the present time; distance moduli obtained by different authors differ quite significantly. Based on an analysis of a variety of distance indicators, van den Bergh (1992) found a distance modulus of $26.0 \mathrm{mag}$, while Freedman et al. (1992) obtained a value of $26.66 \mathrm{mag}$, using $B V R I$ CCD observations of Cepheids in this galaxy. If we adopt the latter value, our brightness cutoff corresponds to an absolute magnitude of $M_{v}=-4.7 \mathrm{mag}$. In Fig. 1 we present the $B-V$ color-magnitude diagram (CMD) from about 14000 stars observed in the field of NGC 300, with an indication of the location of the selected blue stars.

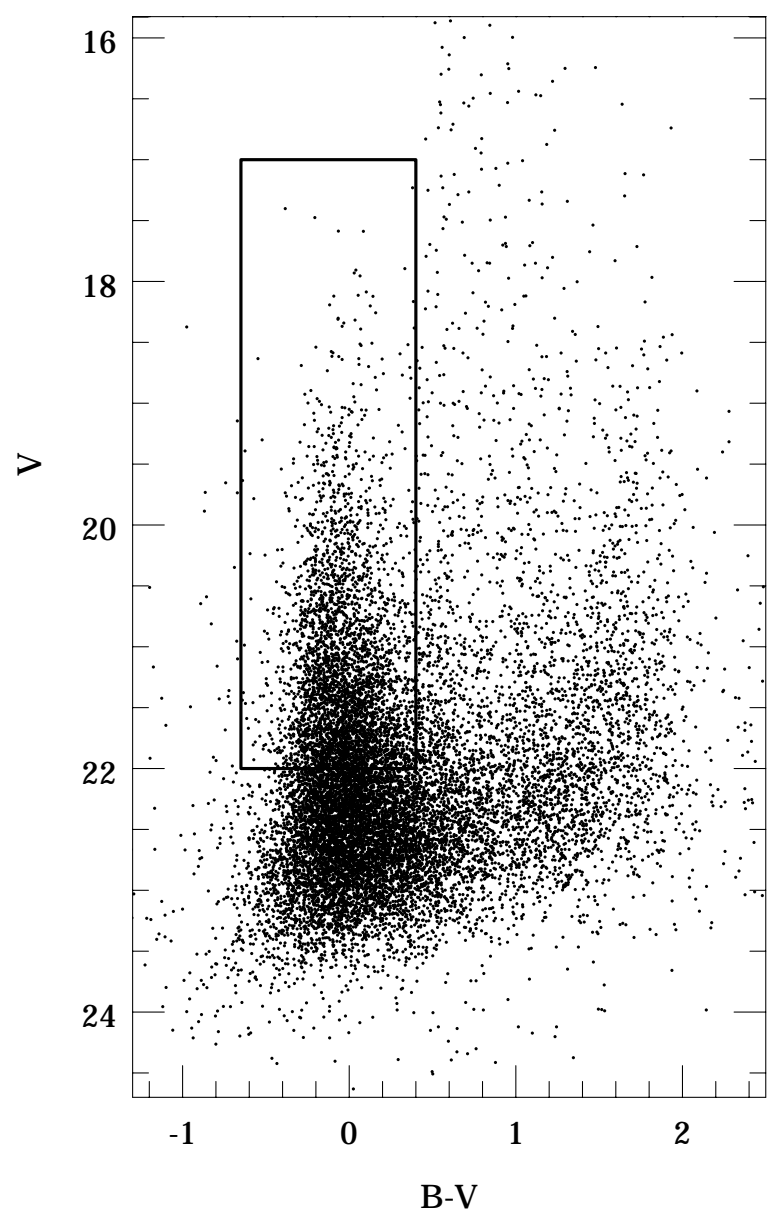

Fig. 1. CMD diagram for about 14000 stars observed in the field of NGC 300. The rectangular region contains blue stars used for searching for OB associations

The next step was to derive the value of $d_{\mathrm{s}}$ based on our catalog of blue stars. To do this we apply the PLC technique with several values for the distance parameter and several values for the minimum number of stars to define an association, $p$. The results are presented in Fig. 2. It can be seen that the position of the maximum of the function $f_{p}(d)$ depends slightly on $p$ and is located in the interval from about 35 to 40 microradians. In order to check on the influence of our choice for the value of $d_{\mathrm{s}}$ on the number of associations we find, we used three search distances, namely $d_{\mathrm{s}}=35,37.5$ and 40 microradians which correspond to linear distances of 72,78 and $83 \mathrm{pc}$, respectively (assuming a distance modulus of $26.66 \mathrm{mag}$ ), and then compared the results. We found that all but three objects were common in all trials. This is because in most cases we can just add a few (10-15\%) new stars to already detected associations if we use a slightly larger value of $d_{\mathrm{s}}$. As a conclusion, the number of detected OB associations is very insensitive to the adopted value of the search radius, in the range suggested by Fig. 2. We adopted $d_{\mathrm{s}}=$ 37.5 microradians as the most appropriate search radius.

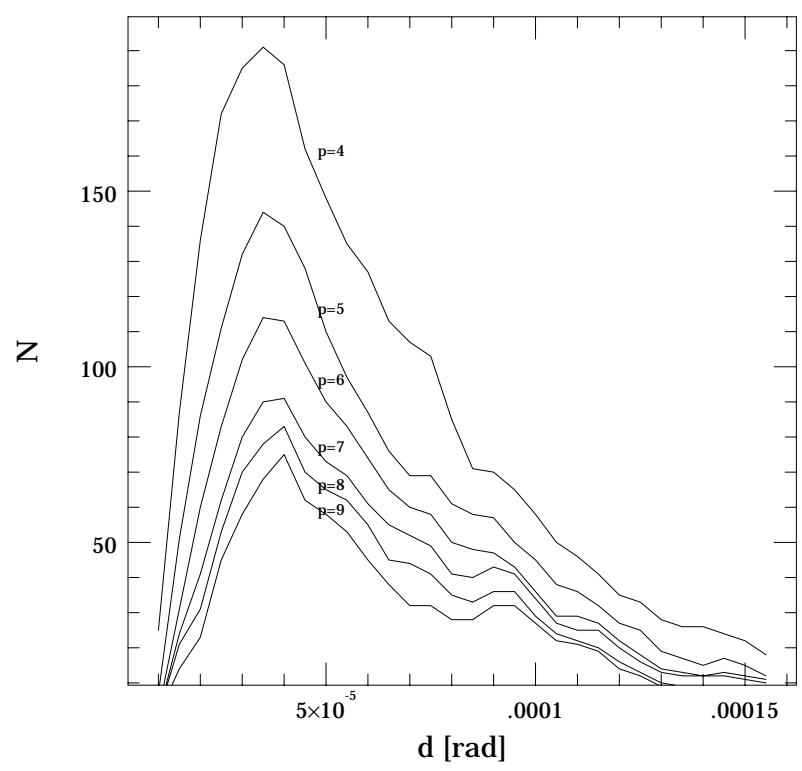

Fig. 2. Behaviour of the function $f_{p}(d)$ for a set of $p$ (minimum number of stars in an association) values

The last parameter that needs to be specified is the minimum number of stars $p$ any potential association should possess. It is clear that too small a number of stars could produce many spurious detections. On the other hand, if we demand an unreasonably large number of stars many small association may be lost. We are then at the tradeoff between completeness and reliability of our sample of associations. In order to check how many detections are caused by chance coincidence, as a function of $p$, a statistical test was performed. One hundred random distributions of a number of stars equal to the number of our sample of blue stars, and distributed over the same area, were created and the PLC technique with our adopted distance parameter value was applied to search for potential groups. This experiment was repeated for different minimum numbers of stars. The plot of the resulting fraction of the spurious detections to the total number of detected associations, as a function of the minimum number of stars, is presented in Fig. 3. We can see that the spurious detections constitute less than $10 \%$ if we adopt 6 stars as a minimum population of OB stars of potential associations. This number seems to be a good compromise and we therefore adopted it. 
Table 1. OB association candidates in NGC 300

\begin{tabular}{|c|c|c|c|c|c|c|}
\hline Name & $\alpha_{2000}$ & $\delta_{2000}$ & $N$ & $\begin{array}{c}\text { size } \\
{[\mu \mathrm{rad}]}\end{array}$ & $\begin{array}{l}\text { size } \\
{[\mathrm{pc}]}\end{array}$ & $\begin{array}{c}\text { cross- } \\
\text { identification }\end{array}$ \\
\hline AS_001 & $0^{\mathrm{h}} 54^{\mathrm{m}} 10^{\mathrm{s}} .9$ & $-37^{\circ} 37^{\prime} 43^{\prime \prime}$ & 6 & 48 & 100 & 3 \\
\hline AS_002 & $0^{\mathrm{h}} 54^{\mathrm{m}} 17^{\mathrm{s}} \cdot 4$ & $-37^{\circ} 35^{\prime} 05^{\prime \prime}$ & 66 & 264 & 552 & $*$ \\
\hline AS_003 & $0^{\mathrm{h}} 54^{\mathrm{m}} 23^{\mathrm{s}} .5$ & $-37^{\circ} 39^{\prime} 53^{\prime \prime}$ & 9 & 78 & 162 & 14 \\
\hline AS_004 & $0^{\mathrm{h}} 54^{\mathrm{m}} 23^{\mathrm{s}} \cdot 6$ & $-37^{\circ} 39^{\prime} 18^{\prime \prime}$ & 12 & 74 & 154 & \\
\hline AS_005 & $0^{\mathrm{h}} 54^{\mathrm{m}} 23^{\mathrm{s}} .7$ & $-37^{\circ} 34^{\prime} 44^{\prime \prime}$ & 15 & 126 & 263 & $12,11,16$ \\
\hline AS_006 & $0^{\mathrm{h}} 54^{\mathrm{m}} 24^{\mathrm{s}} \cdot 4$ & $-37^{\circ} 41^{\prime} 28^{\prime \prime}$ & 10 & 99 & 207 & \\
\hline AS_007 & $0^{\mathrm{h}} 54^{\mathrm{m}} 24^{\mathrm{s}} \cdot 4$ & $-37^{\circ} 38^{\prime} 16^{\prime \prime}$ & 8 & 82 & 172 & \\
\hline AS_008 & $0^{\mathrm{h}} 54^{\mathrm{m}} 25^{\mathrm{s}} .1$ & $-37^{\circ} 35^{\prime} 15^{\prime \prime}$ & 29 & 264 & 552 & \\
\hline AS_009 & $0^{\mathrm{h}} 54^{\mathrm{m}} 25.5$ & $-37^{\circ} 39^{\prime} 34^{\prime \prime}$ & 50 & 208 & 435 & $*$ \\
\hline AS_010 & $0^{\mathrm{h}} 54^{\mathrm{m}} 25^{\mathrm{s}} .9$ & $-37^{\circ} 38^{\prime} 06^{\prime \prime}$ & 14 & 68 & 143 & \\
\hline AS_011 & $0^{\mathrm{h}} 54^{\mathrm{m}} 27^{\mathrm{s}} \cdot 0$ & $-37^{\circ} 43^{\prime} 27^{\prime \prime}$ & 6 & 45 & 94 & 19 \\
\hline AS_012 & $0^{\mathrm{h}} 54^{\mathrm{m}} 27^{\mathrm{s}} .9$ & $-37^{\circ} 39^{\prime} 19^{\prime \prime}$ & 14 & 90 & 188 & \\
\hline AS_013 & $0^{\mathrm{h}} 54^{\mathrm{m}} 28^{\mathrm{s}} \cdot 1$ & $-37^{\circ} 37^{\prime} 44^{\prime \prime}$ & 9 & 55 & 115 & 22 \\
\hline AS_014 & $0^{\mathrm{h}} 54^{\mathrm{m}} 28^{\mathrm{s}} .5$ & $-37^{\circ} 41^{\prime} 32^{\prime \prime}$ & 32 & 148 & 308 & $*$ \\
\hline AS_015 & $0^{\mathrm{h}} 54^{\mathrm{m}} 30^{\mathrm{s}} \cdot 5$ & $-37^{\circ} 43^{\prime} 47^{\prime \prime}$ & 9 & 41 & 87 & 27 \\
\hline AS_016 & $0^{\mathrm{h}} 54^{\mathrm{m}} 31^{\mathrm{s}} 1$ & $-37^{\circ} 37^{\prime} 52^{\prime \prime}$ & 37 & 163 & 341 & $*$ \\
\hline AS_017 & $0^{\mathrm{h}} 54^{\mathrm{m}} 31^{\mathrm{s}} .8$ & $-37^{\circ} 44^{\prime} 12^{\prime \prime}$ & 11 & 82 & 172 & 28,33 \\
\hline AS_018 & $0^{\mathrm{h}} 54^{\mathrm{m}} 31^{\mathrm{s}} .9$ & $-37^{\circ} 38^{\prime} 40^{\prime \prime}$ & 40 & 201 & 420 & $*$ \\
\hline AS_019 & $0^{\mathrm{h}} 54^{\mathrm{m}} 32^{\mathrm{s}} .9$ & $-37^{\circ} 39^{\prime} 11^{\prime \prime}$ & 7 & 52 & 108 & \\
\hline AS_020 & $0^{\mathrm{h}} 54^{\mathrm{m}} 34^{\mathrm{s}} \cdot 2$ & $-37^{\circ} 37^{\prime} 40^{\prime \prime}$ & 6 & 79 & 164 & \\
\hline AS_021 & $0^{\mathrm{h}} 54^{\mathrm{m}} 34^{\mathrm{s}} \cdot 3$ & $-37^{\circ} 45^{\prime} 48^{\prime \prime}$ & 6 & 68 & 142 & 36 \\
\hline AS_022 & $0^{\mathrm{h}} 54^{\mathrm{m}} 34^{\mathrm{s}} \cdot 4$ & $-37^{\circ} 42^{\prime} 54^{\prime \prime}$ & 7 & 47 & 98 & \\
\hline AS_023 & $0^{\mathrm{h}} 54^{\mathrm{m}} 35^{\mathrm{s}} \cdot 2$ & $-37^{\circ} 39^{\prime} 40^{\prime \prime}$ & 7 & 41 & 86 & 37,38 \\
\hline AS_024 & $0^{\mathrm{h}} 54^{\mathrm{m}} 35^{\mathrm{s}} .9$ & $-37^{\circ} 41^{\prime} 08^{\prime \prime}$ & 8 & 74 & 156 & \\
\hline AS_025 & $0^{\mathrm{h}} 54^{\mathrm{m}} 38^{\mathrm{s}} .8$ & $-37^{\circ} 41^{\prime} 36^{\prime \prime}$ & 10 & 97 & 204 & 39,41 \\
\hline AS_026 & $0^{\mathrm{h}} 54^{\mathrm{m}} 39^{\mathrm{s}} .7$ & $-37^{\circ} 42^{\prime} 45^{\prime \prime}$ & 34 & 197 & 411 & $*$ \\
\hline AS_027 & $0^{\mathrm{h}} 54^{\mathrm{m}} 39^{\mathrm{s}} .8$ & $-37^{\circ} 40^{\prime} 21^{\prime \prime}$ & 37 & 150 & 314 & $*$ \\
\hline AS_028 & $0^{\mathrm{h}} 54^{\mathrm{m}} 40^{\mathrm{s}} \cdot 1$ & $-37^{\circ} 39^{\prime} 27^{\prime \prime}$ & 6 & 55 & 116 & \\
\hline AS_029 & $0^{\mathrm{h}} 54^{\mathrm{m}} 41^{\mathrm{s}} \cdot 1$ & $-37^{\circ} 40^{\prime} 54^{\prime \prime}$ & 42 & 219 & 459 & $*$ \\
\hline AS_030 & $0^{\mathrm{h}} 54^{\mathrm{m}} 41^{\mathrm{s}} \cdot 4$ & $-37^{\circ} 35^{\prime} 46^{\prime \prime}$ & 11 & 88 & 184 & 47 \\
\hline AS_031 & $0^{\mathrm{h}} 54^{\mathrm{m}} 41.7$ & $-37^{\circ} 41^{\prime} 48^{\prime \prime}$ & 24 & 193 & 404 & \\
\hline AS_032 & $0^{\mathrm{h}} 54^{\mathrm{m}} 41^{\mathrm{s}} .9$ & $-37^{\circ} 38^{\prime} 33^{\prime \prime}$ & 6 & 68 & 143 & 48 \\
\hline AS_033 & $0^{\mathrm{h}} 54^{\mathrm{m}} 42^{\mathrm{s}} .1$ & $-37^{\circ} 39^{\prime} 04^{\prime \prime}$ & 10 & 67 & 139 & 49,50 \\
\hline AS_034 & $0^{\mathrm{h}} 54^{\mathrm{m}} 42^{\mathrm{s}} \cdot 6$ & $-37^{\circ} 43^{\prime} 06^{\prime \prime}$ & 25 & 110 & 230 & $53 \mathrm{~A}, 53 \mathrm{~B}, 53 \mathrm{C}$ \\
\hline AS_035 & $0^{\mathrm{h}} 54^{\mathrm{m}} 42^{\mathrm{s}} .7$ & $-37^{\circ} 40^{\prime} 15^{\prime \prime}$ & 6 & 42 & 88 & 51 \\
\hline AS_036 & $0^{\mathrm{h}} 54^{\mathrm{m}} 42^{\mathrm{s}} .7$ & $-37^{\circ} 41^{\prime} 21^{\prime \prime}$ & 20 & 110 & 229 & \\
\hline AS_037 & $0^{\mathrm{h}} 54^{\mathrm{m}} 42^{\mathrm{s}} .8$ & $-37^{\circ} 40^{\prime} 03^{\prime \prime}$ & 12 & 79 & 165 & 51,52 \\
\hline AS_038 & $0^{\mathrm{h}} 54^{\mathrm{m}} 43^{\mathrm{s}} \cdot 2$ & $-37^{\circ} 43^{\prime} 41^{\prime \prime}$ & 6 & 79 & 165 & \\
\hline AS_039 & $0^{\mathrm{h}} 54^{\mathrm{m}} 43^{\mathrm{s}} \cdot 3$ & $-37^{\circ} 39^{\prime} 12^{\prime \prime}$ & 7 & 53 & 110 & \\
\hline AS_040 & $0^{\mathrm{h}} 54^{\mathrm{m}} 43^{\mathrm{s}} \cdot 6$ & $-37^{\circ} 45^{\prime} 33^{\prime \prime}$ & 16 & 108 & 225 & \\
\hline AS_041 & $0^{\mathrm{h}} 54^{\mathrm{m}} 43^{\mathrm{s}} \cdot 6$ & $-37^{\circ} 40^{\prime} 41^{\prime \prime}$ & 8 & 74 & 155 & \\
\hline AS_042 & $0^{\mathrm{h}} 54^{\mathrm{m}} 43^{\mathrm{s}} \cdot 6$ & $-37^{\circ} 38^{\prime} 37^{\prime \prime}$ & 7 & 73 & 153 & \\
\hline AS_043 & $0^{\mathrm{h}} 54^{\mathrm{m}} 44^{\mathrm{s}} .7$ & $-37^{\circ} 42^{\prime} 01^{\prime \prime}$ & 6 & 53 & 110 & \\
\hline AS_044 & $0^{\mathrm{h}} 54^{\mathrm{m}} 44^{\mathrm{s}} .9$ & $-37^{\circ} 41^{\prime} 06^{\prime \prime}$ & 14 & 95 & 199 & 60 \\
\hline AS_045 & $0^{\mathrm{h}} 54^{\mathrm{m}} 45^{\mathrm{s}} \cdot 4$ & $-37^{\circ} 38^{\prime} 45^{\prime \prime}$ & 8 & 63 & 132 & 61,62 \\
\hline AS_046 & $0^{\mathrm{h}} 54^{\mathrm{m}} 45^{\mathrm{s}} .9$ & $-37^{\circ} 40^{\prime} 26^{\prime \prime}$ & 57 & 221 & 462 & 56,64 \\
\hline AS_047 & $0^{\mathrm{h}} 54^{\mathrm{m}} 46^{\mathrm{s}} \cdot 4$ & $-37^{\circ} 42^{\prime} 12^{\prime \prime}$ & 13 & 92 & 193 & \\
\hline AS_048 & $0^{\mathrm{h}} 54^{\mathrm{m}} 47^{\mathrm{s}} \cdot 2$ & $-37^{\circ} 38^{\prime} 03^{\prime \prime}$ & 16 & 95 & 199 & 66,68 \\
\hline AS_049 & $0^{\mathrm{h}} 54^{\mathrm{m}} 48^{\mathrm{s}} .1$ & $-37^{\circ} 43^{\prime} 06^{\prime \prime}$ & 11 & 78 & 162 & \\
\hline AS_050 & $0^{\mathrm{h}} 54^{\mathrm{m}} 49^{\mathrm{s}} \cdot 1$ & $-37^{\circ} 39^{\prime} 48^{\prime \prime}$ & 7 & 62 & 129 & 70 \\
\hline AS_051 & $0^{\mathrm{h}} 54^{\mathrm{m}} 49^{\mathrm{s}} .5$ & $-37^{\circ} 33^{\prime} 25^{\prime \prime}$ & 6 & 31 & 64 & 72 \\
\hline AS_052 & $0^{\mathrm{h}} 54^{\mathrm{m}} 49^{\mathrm{s}} \cdot 6$ & $-37^{\circ} 38^{\prime} 48^{\prime \prime}$ & 101 & 323 & 675 & $*, \mathrm{I}$ \\
\hline
\end{tabular}

Column 7: Arabic and roman numbers correspond to H II regions (Deharveng et al. 1988), and objects studied by Breysacher et al. (1997), respectively.

* Means that more detailed cross-identification is given in Table 3. 
Table 1. continued

\begin{tabular}{|c|c|c|c|c|c|c|}
\hline Name & $\alpha_{2000}$ & $\delta_{2000}$ & $N$ & $\begin{array}{c}\text { size } \\
{[\mu \mathrm{rad}]}\end{array}$ & $\begin{array}{l}\text { size } \\
{[\mathrm{pc}]}\end{array}$ & $\begin{array}{c}\text { cross- }^{-} \\
\text {identification }\end{array}$ \\
\hline AS_053 & $0^{\mathrm{h}} 54^{\mathrm{m}} 49^{\mathrm{s}} .8$ & $-37^{\circ} 41^{\prime} 01^{\prime \prime}$ & 25 & 101 & 212 & 74,80 \\
\hline AS_054 & $0^{\mathrm{h}} 54^{\mathrm{m}} 50^{\mathrm{s}} \cdot 1$ & $-37^{\circ} 35^{\prime} 51^{\prime \prime}$ & 6 & 52 & 109 & \\
\hline AS_055 & $0^{\mathrm{h}} 54^{\mathrm{m}} 50^{\mathrm{s}} 6$ & $-37^{\circ} 36^{\prime} 21^{\prime \prime}$ & 6 & 64 & 134 & \\
\hline AS_056 & $0^{\mathrm{h}} 54^{\mathrm{m}} 50^{\mathrm{s}} \cdot 7$ & $-37^{\circ} 40^{\prime} 24^{\prime \prime}$ & 55 & 203 & 424 & $*$ \\
\hline AS_057 & $0^{\mathrm{h}} 54^{\mathrm{m}} 51^{\mathrm{s}} .4$ & $-37^{\circ} 39^{\prime} 44^{\prime \prime}$ & 6 & 58 & 121 & 83,84 \\
\hline AS_058 & $0^{\mathrm{h}} 54^{\mathrm{m}} 51^{\mathrm{s}} .4$ & $-37^{\circ} 41^{\prime} 43^{\prime \prime}$ & 34 & 180 & 376 & $*$ \\
\hline AS_059 & $0^{\mathrm{h}} 54^{\mathrm{m}} 52^{\mathrm{s}} .5$ & $-37^{\circ} 38^{\prime} 53^{\prime \prime}$ & 14 & 130 & 271 & \\
\hline AS_060 & $0^{\mathrm{h}} 54^{\mathrm{m}} 53^{\mathrm{s}} \cdot 0$ & $-37^{\circ} 43^{\prime} 45^{\prime \prime}$ & 22 & 115 & 241 & $87,88,90,91,93$ \\
\hline AS_061 & $0^{\mathrm{h}} 54^{\mathrm{m}} 53^{\mathrm{s}} \cdot 2$ & $-37^{\circ} 40^{\prime} 08^{\prime \prime}$ & 11 & 65 & 136 & \\
\hline AS_062 & $0^{\mathrm{h}} 54^{\mathrm{m}} 54^{\mathrm{s}} \cdot 0$ & $-37^{\circ} 35^{\prime} 48^{\prime \prime}$ & 6 & 63 & 132 & \\
\hline AS_063 & $0^{\mathrm{h}} 54^{\mathrm{m}} 54^{\mathrm{s}} .7$ & $-37^{\circ} 36^{\prime} 49^{\prime \prime}$ & 23 & 113 & 236 & 92,94 \\
\hline AS_064 & $0^{\mathrm{h}} 54^{\mathrm{m}} 55^{\mathrm{s}} 5$ & $-37^{\circ} 45^{\prime} 44^{\prime \prime}$ & 17 & 137 & 287 & 97 \\
\hline AS_065 & $0^{\mathrm{h}} 54^{\mathrm{m}} 55^{\mathrm{s}} .9$ & $-37^{\circ} 40^{\prime} 13^{\prime \prime}$ & 9 & 23 & 48 & 96 \\
\hline AS_066 & $0^{\mathrm{h}} 54^{\mathrm{m}} 56^{\mathrm{s}} \cdot 1$ & $-37^{\circ} 44^{\prime} 33^{\prime \prime}$ & 8 & 72 & 150 & \\
\hline AS_067 & $0^{\mathrm{h}} 54^{\mathrm{m}} 56^{\mathrm{s}} .5$ & $-37^{\circ} 41^{\prime} 11^{\prime \prime}$ & 13 & 81 & 169 & 100 \\
\hline AS_068 & $0^{\mathrm{h}} 54^{\mathrm{m}} 57^{\mathrm{s}} \cdot 1$ & $-37^{\circ} 40^{\prime} 35^{\prime \prime}$ & 9 & 82 & 172 & 98 \\
\hline AS_069 & $0^{\mathrm{h}} 54^{\mathrm{m}} 57^{\mathrm{s}} \cdot 2$ & $-37^{\circ} 41^{\prime} 34^{\prime \prime}$ & 8 & 68 & 141 & \\
\hline AS_070 & $0^{\mathrm{h}} 54^{\mathrm{m}} 57^{\mathrm{s}} .8$ & $-37^{\circ} 45^{\prime} 43^{\prime \prime}$ & 6 & 36 & 75 & \\
\hline AS_071 & $0^{\mathrm{h}} 54^{\mathrm{m}} 57^{\mathrm{s}} \cdot 9$ & $-37^{\circ} 42^{\prime} 32^{\prime \prime}$ & 10 & 110 & 229 & 103,104 \\
\hline AS_072 & $0^{\mathrm{h}} 54^{\mathrm{m}} 58^{\mathrm{s}} \cdot 3$ & $-37^{\circ} 41^{\prime} 11^{\prime \prime}$ & 6 & 65 & 136 & 105 \\
\hline AS_073 & $0^{\mathrm{h}} 54^{\mathrm{m}} 58^{\mathrm{s}} .8$ & $-37^{\circ} 44^{\prime} 19^{\prime \prime}$ & 9 & 85 & 177 & 108 \\
\hline AS_074 & $0^{\mathrm{h}} 54^{\mathrm{m}} 59^{\mathrm{s}} 0$ & $-37^{\circ} 36^{\prime} 34^{\prime \prime}$ & 12 & 53 & 111 & \\
\hline AS_075 & $0^{\mathrm{h}} 54^{\mathrm{m}} 59^{\mathrm{s}} .1$ & $-37^{\circ} 43^{\prime} 20^{\prime \prime}$ & 9 & 84 & 176 & \\
\hline AS_076 & $0^{\mathrm{h}} 55^{\mathrm{m}} 00^{\mathrm{s}} \cdot 2$ & $-37^{\circ} 40^{\prime} 41^{\prime \prime}$ & 12 & 50 & 104 & 109 \\
\hline AS_077 & $0^{\mathrm{h}} 55^{\mathrm{m}} 01^{\mathrm{s}} \cdot 2$ & $-37^{\circ} 39^{\prime} 29^{\prime \prime}$ & 6 & 62 & 129 & \\
\hline AS_078 & $0^{\mathrm{h}} 55^{\mathrm{m}} 01^{\mathrm{s}} .7$ & $-37^{\circ} 42^{\prime} 22^{\prime \prime}$ & 6 & 28 & 58 & \\
\hline AS_079 & $0^{\mathrm{h}} 55^{\mathrm{m}} 01^{\mathrm{s}} .9$ & $-37^{\circ} 41^{\prime} 49^{\prime \prime}$ & 6 & 70 & 146 & \\
\hline AS_080 & $0^{\mathrm{h}} 55^{\mathrm{m}} 02^{\mathrm{s}} .1$ & $-37^{\circ} 36^{\prime} 53^{\prime \prime}$ & 8 & 48 & 100 & \\
\hline AS_081 & $0^{\mathrm{h}} 55^{\mathrm{m}} 02^{\mathrm{s}} .3$ & $-37^{\circ} 44^{\prime} 26^{\prime \prime}$ & 12 & 65 & 136 & 113,116 \\
\hline AS_082 & $0^{\mathrm{h}} 55^{\mathrm{m}} 02^{\mathrm{s}} .6$ & $-37^{\circ} 38^{\prime} 25^{\prime \prime}$ & 40 & 139 & 291 & 115 \\
\hline AS_083 & $0^{\mathrm{h}} 55^{\mathrm{m}} 02^{\mathrm{s}} .9$ & $-37^{\circ} 40^{\prime} 04^{\prime \prime}$ & 11 & 53 & 111 & \\
\hline AS_084 & $0^{\mathrm{h}} 55^{\mathrm{m}} 03^{\mathrm{s}} .1$ & $-37^{\circ} 42^{\prime} 47^{\prime \prime}$ & 62 & 227 & 475 & $118 \mathrm{~A}, \mathrm{III}$ \\
\hline AS_085 & $0^{\mathrm{h}} 55^{\mathrm{m}} 03^{\mathrm{s}} \cdot 3$ & $-37^{\circ} 41^{\prime} 21^{\prime \prime}$ & 13 & 121 & 252 & 123 \\
\hline AS_086 & $0^{\mathrm{h}} 55^{\mathrm{m}} 03.5$ & $-37^{\circ} 43^{\prime} 23^{\prime \prime}$ & 30 & 119 & 248 & 119A, 119B, II \\
\hline AS_087 & $0^{\mathrm{h}} 55^{\mathrm{m}} 03^{\mathrm{s}} .8$ & $-37^{\circ} 40^{\prime} 29^{\prime \prime}$ & 6 & 54 & 112 & \\
\hline AS_088 & $0^{\mathrm{h}} 55^{\mathrm{m}} 04^{\mathrm{s}} \cdot 2$ & $-37^{\circ} 39^{\prime} 26^{\prime \prime}$ & 16 & 133 & 279 & 120 \\
\hline AS_089 & $0^{\mathrm{h}} 55^{\mathrm{m}} 04^{\mathrm{s}} \cdot 2$ & $-37^{\circ} 41^{\prime} 14^{\prime \prime}$ & 6 & 33 & 70 & \\
\hline AS_090 & $0^{\mathrm{h}} 55^{\mathrm{m}} 04^{\mathrm{s}} .5$ & $-37^{\circ} 40^{\prime} 53^{\prime \prime}$ & 16 & 67 & 139 & 122 \\
\hline AS_091 & $0^{\mathrm{h}} 55^{\mathrm{m}} 05^{\mathrm{s}} \cdot 0$ & $-37^{\circ} 41^{\prime} 44^{\prime \prime}$ & 7 & 78 & 163 & 123 \\
\hline AS_092 & $0^{\mathrm{h}} 55^{\mathrm{m}} 05^{\mathrm{s}} 3$ & $-37^{\circ} 41^{\prime} 26^{\prime \prime}$ & 8 & 49 & 103 & 123,124 \\
\hline AS_093 & $0^{\mathrm{h}} 55^{\mathrm{m}} 05^{\mathrm{s}} .9$ & $-37^{\circ} 43^{\prime} 14^{\prime \prime}$ & 9 & 99 & 207 & 119B \\
\hline AS_094 & $0^{\mathrm{h}} 55^{\mathrm{m}} 06^{\mathrm{s}} .9$ & $-37^{\circ} 41^{\prime} 05^{\prime \prime}$ & 14 & 80 & 168 & 126 \\
\hline AS_095 & $0^{\mathrm{h}} 55^{\mathrm{m}} 07^{\mathrm{s}} \cdot 6$ & $-37^{\circ} 42^{\prime} 58^{\prime \prime}$ & 7 & 55 & 114 & \\
\hline AS_096 & $0^{\mathrm{h}} 55^{\mathrm{m}} 08^{\mathrm{s}} \cdot 4$ & $-37^{\circ} 40^{\prime} 21^{\prime \prime}$ & 33 & 187 & 392 & 130 \\
\hline AS_097 & $0^{\mathrm{h}} 55^{\mathrm{m}} 08^{\mathrm{s}} .7$ & $-37^{\circ} 41^{\prime} 58^{\prime \prime}$ & 7 & 80 & 168 & 127 \\
\hline AS_098 & $0^{\mathrm{h}} 55^{\mathrm{m}} 08^{\mathrm{s}} .8$ & $-37^{\circ} 40^{\prime} 50^{\prime \prime}$ & 6 & 41 & 86 & 130 \\
\hline AS_099 & $0^{\mathrm{h}} 55^{\mathrm{m}} 08^{\mathrm{s}} .8$ & $-37^{\circ} 39^{\prime} 33^{\prime \prime}$ & 13 & 71 & 148 & 129 \\
\hline AS_100 & $0^{\mathrm{h}} 55^{\mathrm{m}} 09^{\mathrm{s}} \cdot 3$ & $-37^{\circ} 47^{\prime} 56^{\prime \prime}$ & 10 & 45 & 95 & 131,133 \\
\hline AS_101 & $0^{\mathrm{h}} 55^{\mathrm{m}} 12^{\mathrm{s}} \cdot 6$ & $-37^{\circ} 44^{\prime} 12^{\prime \prime}$ & 33 & 177 & 369 & $*$ \\
\hline AS_102 & $0^{\mathrm{h}} 55^{\mathrm{m}} 12^{\mathrm{s}} .7$ & $-37^{\circ} 41^{\prime} 31^{\prime \prime}$ & 63 & 174 & 363 & * \\
\hline AS_103 & $0^{\mathrm{h}} 55^{\mathrm{m}} 13^{\mathrm{s}} \cdot 4$ & $-37^{\circ} 37^{\prime} 47^{\prime \prime}$ & 6 & 36 & 75 & \\
\hline AS_104 & $0^{\mathrm{h}} 55^{\mathrm{m}} 14^{\mathrm{s}} \cdot 2$ & $-37^{\circ} 40^{\prime} 16^{\prime \prime}$ & 6 & 49 & 103 & \\
\hline AS_105 & $0^{\mathrm{h}} 55^{\mathrm{m}} 14^{\mathrm{s}} \cdot 2$ & $-37^{\circ} 41^{\prime} 16^{\prime \prime}$ & 6 & 49 & 102 & \\
\hline AS_106 & $0^{\mathrm{h}} 55^{\mathrm{m}} 15^{\mathrm{s}} \cdot 1$ & $-37^{\circ} 42^{\prime} 05^{\prime \prime}$ & 6 & 37 & 77 & \\
\hline
\end{tabular}

Column 7: Arabic and roman numbers correspond to H II regions (Deharveng et al. 1988), and objects studied by Breysacher et al. (1997), respectively.

* Means that more detailed cross-identification is given in Table 3. 
Table 1. continued

\begin{tabular}{ccccccc}
\hline Name & $\alpha_{2000}$ & $\delta_{2000}$ & $N$ & $\begin{array}{c}\text { size } \\
{[\mu \mathrm{rad}]}\end{array}$ & $\begin{array}{c}\text { size } \\
{[\mathrm{pc}]}\end{array}$ & $\begin{array}{c}\text { cross- } \\
\text { identification }\end{array}$ \\
\hline AS_107 & $0^{\mathrm{h}} 55^{\mathrm{m}} 15^{\mathrm{s}} 6$ & $-37^{\circ} 44^{\prime} 30^{\prime \prime}$ & 15 & 101 & 210 & $140,141,142$ \\
AS_108 & $0^{\mathrm{h}} 55^{\mathrm{m}} 19.2$ & $-37^{\circ} 44^{\prime} 09^{\prime \prime}$ & 10 & 83 & 174 & \\
AS_109 & $0^{\mathrm{h}} 55^{\mathrm{m}} 20.7$ & $-37^{\circ} 43^{\prime} 49^{\prime \prime}$ & 17 & 141 & 294 & 145,146 \\
AS_110 & $0^{\mathrm{h}} 55^{\mathrm{m}} 24^{\mathrm{s}} 5$ & $-37^{\circ} 39^{\prime} 32^{\prime \prime}$ & 11 & 66 & 139 & 147 \\
AS_111 & $0^{\mathrm{h}} 55^{\mathrm{m}} 30^{\mathrm{s}} 2$ & $-37^{\circ} 39^{\prime} 11^{\prime \prime}$ & 12 & 93 & 194 & 153,154 \\
AS_112 & $0^{\mathrm{h}} 55^{\mathrm{m}} 30^{\mathrm{s}} .9$ & $-37^{\circ} 36^{\prime} 05^{\prime \prime}$ & 6 & 11 & 24 & \\
AS_113 & $0^{\mathrm{h}} 55^{\mathrm{m}} 33^{\mathrm{s}} 4$ & $-37^{\circ} 43^{\prime} 16^{\prime \prime}$ & 11 & 66 & 137 & 159 \\
AS_114 & $0^{\mathrm{h}} 55^{\mathrm{m}} 33^{\mathrm{s}} .8$ & $-37^{\circ} 41^{\prime} 15^{\prime \prime}$ & 11 & 63 & 131 & \\
AS_115 & $0^{\mathrm{h}} 55^{\mathrm{m}} 36.0$ & $-37^{\circ} 41^{\prime} 50^{\prime \prime}$ & 9 & 62 & 130 & 161 \\
AS_116 & $0^{\mathrm{h}} 55^{\mathrm{m}} 36.2$ & $-37^{\circ} 41^{\prime} 29^{\prime \prime}$ & 10 & 90 & 189 & \\
AS_117 & $0^{\mathrm{h}} 55^{\mathrm{m}} 38^{\mathrm{s}} 2$ & $-37^{\circ} 44^{\prime} 03^{\prime \prime}$ & 8 & 66 & 138 & \\
\hline
\end{tabular}

Column 7: Arabic and roman numbers correspond to H II regions (Deharveng et al. 1988), and objects studied by Breysacher et al. (1997), respectively.

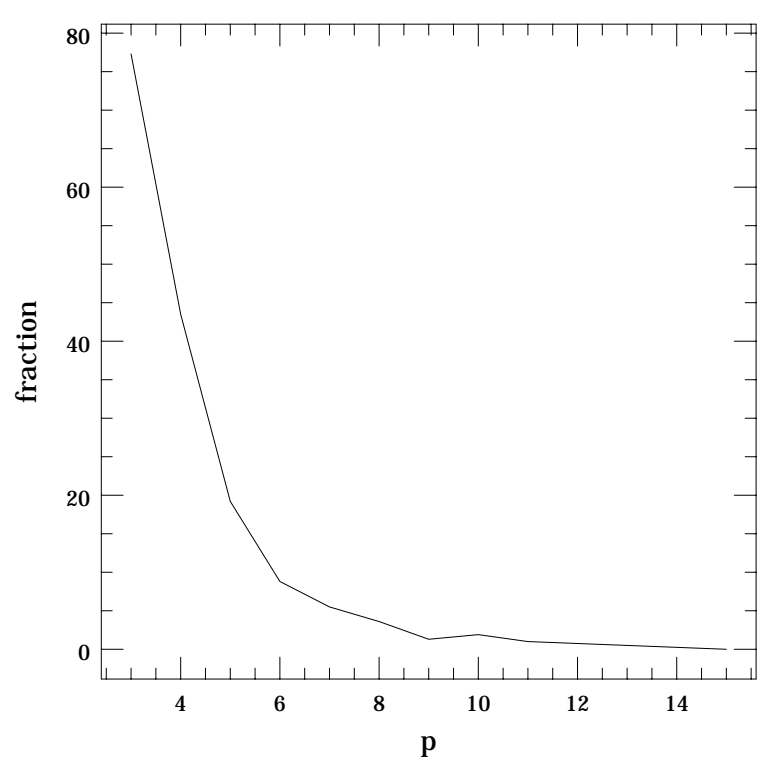

Fig. 3. The percentage of expected spurious detections of associations, versus $p$, the minimum number of stars in an association

\section{Results}

\subsection{The catalog}

The application of the PLC technique, with a threshold of number of stars equal to 6 and the distance parameter value of 37.5 microradians as discussed above, resulted in the detection of 117 groups of blue stars. Table 1 contains their description. Columns 1-3 give the designation, and the equatorial coordinates of the detected associations. The number of blue stars belonging to the group, its size in both microradians and parsecs (distance modulus of 26.66 assumed), cross-identification with the catalog of H II regions in NGC 300 (Deharveng et al. 1988) and objects studied by Breysacher et al. (1997), (arabic and roman numbers respectively) are given in the following columns.

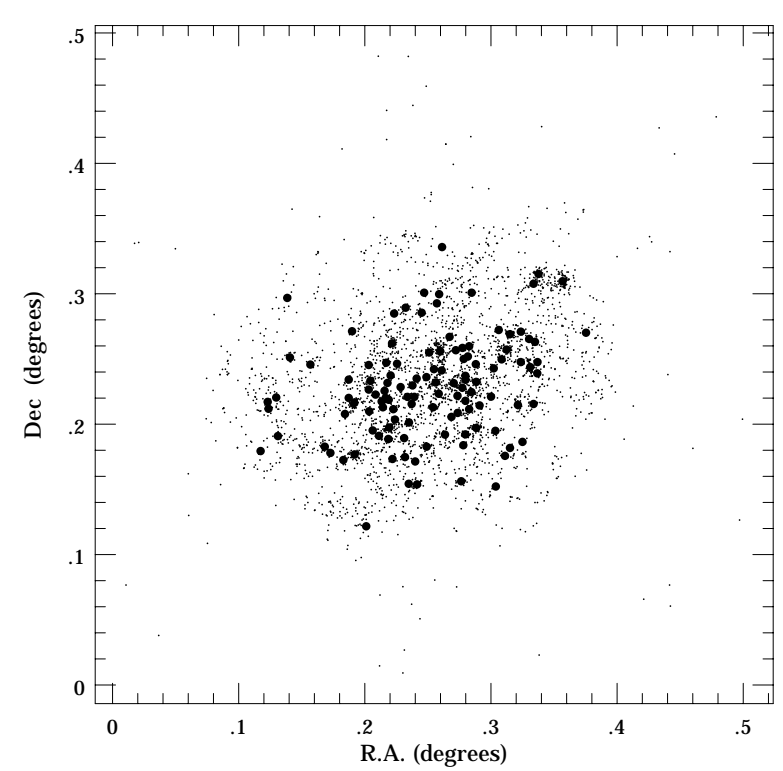

Fig. 4. Spatial distribution of detected OB associations (filled circles) over the disc of NGC 300. Dots are individual blue stars. The center of the figure corresponds to $\alpha_{2000}=$ $0^{\mathrm{h}} 54^{\mathrm{s}} 4, \delta_{2000}=-37^{\circ} 41^{\prime} 03^{\prime \prime}$

The coordinates of the OB associations were determined as the mean coordinates of their separate member stars. Following Battinelli (1991), the real diameter of associations is defined as $0.5 *\left(\Phi_{\mathrm{RA}} * \cos \left(\Phi_{\text {Dec }}\right)+\Phi_{\text {Dec }}\right) *$ $d_{\mathrm{NGC} 300}$, where $\Phi_{\mathrm{RA}}$ and $\Phi_{\mathrm{Dec}}$ correspond to the diameter in right ascention and declination respectively and $d_{\text {NGC } 300}$ stands for distance to the galaxy. We assumed $d_{\mathrm{NGC} 300}=2.1 \mathrm{Mpc}$ (distance modulus of $26.66 \mathrm{mag}$ ). Figure 4 displays the spatial distribution of the detected associations over the disc of NGC 300. Maps of six typical associations are presented in the Appendix.

We would like to stress that our spatial resolution allows detection of associations as small as about 15 pc. We therefore expect that our catalog is near complete even down to potentially very compact associations. 


\subsection{Size distribution}

In Fig. 5 we show the size distribution of the detected associations. There is a sharp peak located at about 60 microradians. This value depends somewhat on the assumed value of $d_{\mathrm{s}}$. Using $d_{\mathrm{s}}=35$ and $d_{\mathrm{s}}=40$ microradians, respectively, sizes are about $15 \%$ different (smaller and bigger, respectively), but the shape of the distribution remains the same. Table 2 gives numerical values for the location of the peak of the size distribution, for a range of distance parameters, and for the two different distance moduli to NGC 300 discussed before.

Until a more precise distance to NGC 300 is known (which is one of the main purposes of our NGC 300 project), it is not possible to carry out an unambiguous comparison of our size results with those obtained for $\mathrm{OB}$ associations in other galaxies.

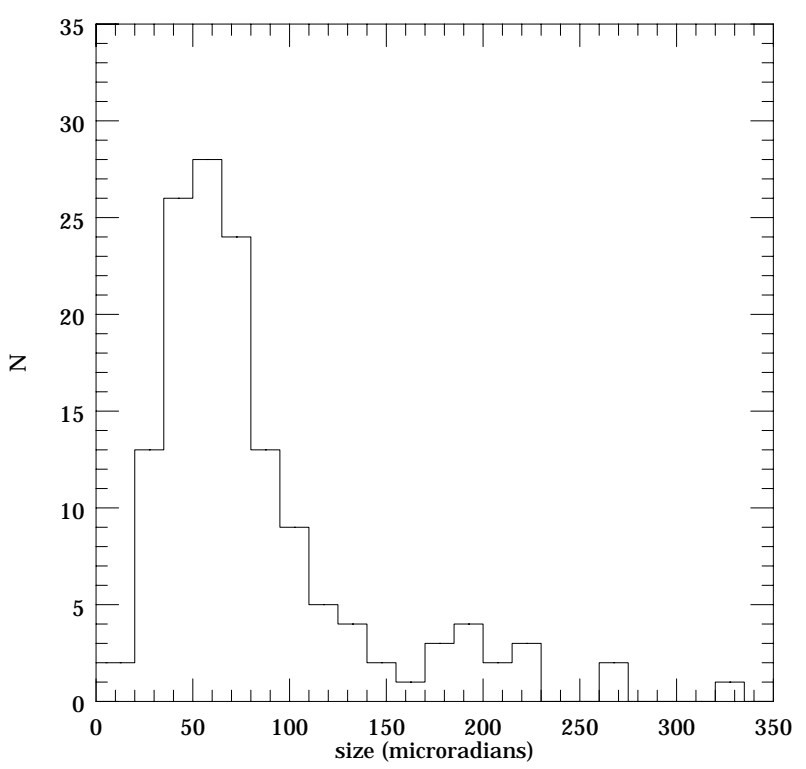

Fig. 5. Size distribution of associations in NGC 300

\subsection{Correlation with $\mathrm{H}$ II regions}

In most cases the ionization of hydrogen is caused by UV photons emitted from nearby hot, massive stars, so one can suspect a correlation of the spatial distributions of OB associations and H II regions. To check this we compared the spatial distribution of OB associations obtained in this paper with the spatial distribution of $\mathrm{H}$ II regions in NGC 300 cataloged by Deharveng et al. (1988). The result is that about $60 \%$ of the detected $\mathrm{OB}$ associations do clearly overlap with H II regions (see Tables 1 and 3 for detailed cross-identification), which indicates that there is indeed the expected strong correlation. Thus both OB associations and H II regions appear to be about equally well suited tracers of recent or ongoing star formation.

\subsection{Stellar complexes}

We can see from Fig. 5 that besides the stellar groups with sizes typical for OB associations (of about $100 \mathrm{pc}$ )
Table 2. Peak of size distribution

\begin{tabular}{cccc}
\hline peak location & $d_{\mathrm{s}}=35$ & $d_{\mathrm{s}}=37.5$ & $d_{\mathrm{s}}=40$ \\
\hline$\mu \mathrm{rad}$ & 53 & 60 & 69 \\
in pc for $\mu=26.0 \mathrm{mag}$ & 84 & 95 & 110 \\
in pc for $\mu=26.66 \mathrm{mag})$ & 110 & 125 & 144 \\
\hline
\end{tabular}

there are also some groups having much bigger dimensions. Regarding their size they resemble Galactic star complexes, as described by Efremov et al. (1987).

By visual inspection of the objects identified as having sizes larger than 300 pc (potential star complexes) we found that most of them appear to consist of a number of smaller, well defined associations with sizes close to $100 \mathrm{pc}$ (see the map of one of the stellar complexes presented in the Appendix). These were not detected as individual stellar groupings by the PLC technique due to the high density of blue stars with respect to the surrounding regions in these parts of the galaxy.

To check on this in an objective way we selected blue stars from each of these regions and applied the PLC method for a second time. In 14 out of 18 cases we detected that the apparently large single associations do indeed consist of several smaller associations. Their description is given in Table 3 . The names of new objects were designated using the name of the corresponding associations detected during the first application of the PLC technique, followed by consecutive roman letters. (e.g. AS_102a, AS_102b, etc.) Their parameters were derived in the same manner as described in Sect. 4.1. As a conclusion, very large single OB associations (like AS_008, AS_031, AS_084 and AS_096) seem to be very rare.

It is interesting to note that 14 out of 18 potential star complexes are located in the western part of NGC 300, while the distribution of detected $\mathrm{OB}$ associations seems to be fairly uniform (see Fig. 4). This fact suggests that for some unknown reasons the physical conditions for the formation of large objects have been more favorable in this part of the galaxy.

\section{Summary}

We have presented the results of a search for OB associations in the spiral galaxy NGC 300, using photometric data obtained within a broader project aiming at a precise distance determination to this galaxy from its Cepheid variables. Magnitudes and colors were obtained for about 14000 stars in a $0.5 \times 0.5$ square degree field centered on the nucleus of NGC 300. From this sample, we selected blue stars using the constraints $V<22$ mag and $-0.6<B-V<0.4$ mag. Application of the PLC method resulted in the detection of 117 groups of blue stars. Statistical considerations show that the expected contamination of this sample by spurious detections is less than $10 \%$. This suggests that our sample constitutes a homogeneous group which is well suited for comparison with systems of associations found in other galaxies. We detected the expected strong correlation between the spatial distributions of associations and $\mathrm{H}$ II regions in 
Table 3. OB association candidates in the NGC 300 found in the regions of potential stellar-complexes

\begin{tabular}{|c|c|c|c|c|c|c|}
\hline Name & $\alpha_{2000}$ & $\delta_{2000}$ & $N$ & $\begin{array}{c}\text { size } \\
{[\mu \mathrm{rad}]}\end{array}$ & $\begin{array}{l}\text { size } \\
{[\mathrm{pc}]} \\
\end{array}$ & $\begin{array}{c}\text { cross- } \\
\text { identification }\end{array}$ \\
\hline AS_002a & $0^{\mathrm{h}} 54^{\mathrm{m}} 16^{\mathrm{s}} \cdot 3$ & $-37^{\circ} 34^{\prime} 57^{\prime \prime}$ & 19 & 55 & 114 & 6 \\
\hline AS_002b & $0^{\mathrm{h}} 54^{\mathrm{m}} 17^{\mathrm{s}} .8$ & $-37^{\circ} 35^{\prime} 07^{\prime \prime}$ & 6 & 26 & 53 & 9 \\
\hline AS_002c & $0^{\mathrm{h}} 54^{\mathrm{m}} 16^{\mathrm{s}} .9$ & $-37^{\circ} 35^{\prime} 18^{\prime \prime}$ & 24 & 131 & 273 & 7,8 \\
\hline AS_002d & $0^{\mathrm{h}} 54^{\mathrm{m}} 17^{\mathrm{s}} \cdot 2$ & $-37^{\circ} 34^{\prime} 46^{\prime \prime}$ & 6 & 26 & 53 & \\
\hline AS_009a & $0^{\mathrm{h}} 54^{\mathrm{m}} 25^{\mathrm{s}} \cdot 3$ & $-37^{\circ} 39^{\prime} 46^{\prime \prime}$ & 20 & 24 & 130 & 15,14 \\
\hline AS_009b & $0^{\mathrm{h}} 54^{\mathrm{m}} 25.6$ & $-37^{\circ} 39^{\prime} 15^{\prime \prime}$ & 14 & 15 & 32 & 17 \\
\hline AS_014a & $0^{\mathrm{h}} 54^{\mathrm{m}} 28^{\mathrm{s}} .5$ & $-37^{\circ} 41^{\prime} 36^{\prime \prime}$ & 17 & 58 & 121 & 24 \\
\hline AS_014b & $0^{\mathrm{h}} 54^{\mathrm{m}} 29.0$ & $-37^{\circ} 41^{\prime} 17^{\prime \prime}$ & 7 & 45 & 93 & \\
\hline AS_016a & $0^{\mathrm{h}} 54^{\mathrm{m}} 30^{\mathrm{s}} .0$ & $-37^{\circ} 37^{\prime} 52^{\prime \prime}$ & 9 & 62 & 129 & \\
\hline AS_016b & $0^{\mathrm{h}} 54^{\mathrm{m}} 31^{\mathrm{s}} \cdot 3$ & $-37^{\circ} 37^{\prime} 54^{\prime \prime}$ & 25 & 108 & 225 & 30,31 \\
\hline AS_018a & $0^{\mathrm{h}} 54^{\mathrm{m}} 30^{\mathrm{s}} \cdot 6$ & $-37^{\circ} 38^{\prime} 39^{\prime \prime}$ & 8 & 49 & 101 & \\
\hline AS_018b & $0^{\mathrm{h}} 54^{\mathrm{m}} 32^{\mathrm{s}} .1$ & $-37^{\circ} 38^{\prime} 43^{\prime \prime}$ & 10 & 54 & 112 & 32,34 \\
\hline AS_026a & $0^{\mathrm{h}} 54^{\mathrm{m}} 38^{\mathrm{s}} \cdot 4$ & $-37^{\circ} 42^{\prime} 41^{\prime \prime}$ & 8 & 26 & 53 & 40 \\
\hline AS_026b & $0^{\mathrm{h}} 54^{\mathrm{m}} 40^{\mathrm{s}} \cdot 2$ & $-37^{\circ} 42^{\prime} 50^{\prime \prime}$ & 15 & 58 & 121 & \\
\hline AS_027a & $0^{\mathrm{h}} 54^{\mathrm{m}} 39^{\mathrm{s}} \cdot 0$ & $-37^{\circ} 40^{\prime} 21^{\prime \prime}$ & 20 & 74 & 153 & \\
\hline AS_027b & $0^{\mathrm{h}} 54^{\mathrm{m}} 40^{\mathrm{s}} \cdot 6$ & $-37^{\circ} 40^{\prime} 20^{\prime \prime}$ & 7 & 32 & 66 & \\
\hline AS_029a & $0^{\mathrm{h}} 54^{\mathrm{m}} 41^{\mathrm{s}} .8$ & $-37^{\circ} 40^{\prime} 59^{\prime \prime}$ & 15 & 70 & 145 & \\
\hline AS_029b & $0^{\mathrm{h}} 54^{\mathrm{m}} 40^{\mathrm{s}} \cdot 3$ & $-37^{\circ} 40^{\prime} 54^{\prime \prime}$ & 8 & 32 & 66 & 45 \\
\hline AS_029c & $0^{\mathrm{h}} 54^{\mathrm{m}} 41^{\mathrm{s}} .1$ & $-37^{\circ} 40^{\prime} 47^{\prime \prime}$ & 6 & 45 & 93 & 45 \\
\hline AS_046a & $0^{\mathrm{h}} 54^{\mathrm{m}} 46^{\mathrm{s}} \cdot 2$ & $-37^{\circ} 40^{\prime} 14^{\prime \prime}$ & 8 & 56 & 115 & 64 \\
\hline AS_046b & $0^{\mathrm{h}} 54^{\mathrm{m}} 46^{\mathrm{s}} \cdot 1$ & $-37^{\circ} 40^{\prime} 31^{\prime \prime}$ & 19 & 114 & 237 & 64 \\
\hline AS_052a & $0^{\mathrm{h}} 54^{\mathrm{m}} 50^{\mathrm{s}} \cdot 2$ & $-37^{\circ} 38^{\prime} 24^{\prime \prime}$ & 9 & 54 & 112 & $77, \mathrm{I}$ \\
\hline AS_052b & $0^{\mathrm{h}} 54^{\mathrm{m}} 49.7$ & $-37^{\circ} 38^{\prime} 38^{\prime \prime}$ & 17 & 64 & 133 & I \\
\hline AS_052c & $0^{\mathrm{h}} 54^{\mathrm{m}} 49^{\mathrm{s}} .5$ & $-37^{\circ} 38^{\prime} 51^{\prime \prime}$ & 6 & 35 & 73 & I \\
\hline AS_052d & $0^{\mathrm{h}} 54^{\mathrm{m}} 51^{\mathrm{s}} .0$ & $-37^{\circ} 38^{\prime} 26^{\prime \prime}$ & 8 & 51 & 106 & $79, \mathrm{I}$ \\
\hline AS_052e & $0^{\mathrm{h}} 54^{\mathrm{m}} 49^{\mathrm{s}} .8$ & $-37^{\circ} 39^{\prime} 20^{\prime \prime}$ & 18 & 70 & 145 & 75,71 \\
\hline AS_056a & $0^{\mathrm{h}} 54^{\mathrm{m}} 49^{\mathrm{s}} \cdot 6$ & $-37^{\circ} 40^{\prime} 03^{\prime \prime}$ & 7 & 43 & 90 & $76 \mathrm{~B}$ \\
\hline AS_056b & $0^{\mathrm{h}} 54^{\mathrm{m}} 49^{\mathrm{s}} .9$ & $-37^{\circ} 40^{\prime} 19^{\prime \prime}$ & 39 & 116 & 241 & $76 \mathrm{~B}$ \\
\hline AS_056c & $0^{\mathrm{h}} 54^{\mathrm{m}} 50.6$ & $-37^{\circ} 40^{\prime} 27^{\prime \prime}$ & 9 & 43 & 89 & $76 \mathrm{~A}, 76 \mathrm{C}$ \\
\hline AS_058a & $0^{\mathrm{h}} 54^{\mathrm{m}} 50^{\mathrm{s}} .7$ & $-37^{\circ} 41^{\prime} 46^{\prime \prime}$ & 10 & 74 & 154 & 81 \\
\hline AS_058b & $0^{\mathrm{h}} 54^{\mathrm{m}} 51^{\mathrm{s}} .8$ & $-37^{\circ} 41^{\prime} 49^{\prime \prime}$ & 13 & 67 & 139 & 81,85 \\
\hline AS_101a & $0^{\mathrm{h}} 55^{\mathrm{m}} 11^{\mathrm{s}} .5$ & $-37^{\circ} 44^{\prime} 06^{\prime \prime}$ & 7 & 38 & 80 & 135 \\
\hline AS_101b & $0^{\mathrm{h}} 55^{\mathrm{m}} 12^{\mathrm{s}} .7$ & $-37^{\circ} 44^{\prime} 15^{\prime \prime}$ & 13 & 68 & 140 & 135,139 \\
\hline AS_102a & $0^{\mathrm{h}} 55^{\mathrm{m}} 12^{\mathrm{s}} .0$ & $-37^{\circ} 41^{\prime} 28^{\prime \prime}$ & 34 & 116 & 241 & $137 \mathrm{~B}, 137 \mathrm{D}$ \\
\hline AS_102b & $0^{\mathrm{h}} 55^{\mathrm{m}} 12^{\mathrm{s}} .8$ & $-37^{\circ} 41^{\prime} 41^{\prime \prime}$ & 20 & 67 & 138 & $137 \mathrm{~A}, 137 \mathrm{~B}$ \\
\hline AS_102c & $0^{\mathrm{h}} 55^{\mathrm{m}} 13^{\mathrm{s}} .7$ & $-37^{\circ} 41^{\prime} 40^{\prime \prime}$ & 13 & 43 & 89 & $137 \mathrm{C}$ \\
\hline
\end{tabular}

Column 7: Arabic and roman numbers correspond to H II regions (Deharveng et al. 1988), and objects studied by Breysacher et al. (1997), respectively.

NGC 300, a fact which lends additional strong support to the relability of our OB association searching procedure.

Most of the detected OB associations have sizes comparable to typical associations from the Galaxy and other Local Group galaxies. However, a few objects have sizes up to five times larger, corresponding rather to stellar complexes than associations. The PLC technique was applied once more to stars from the regions of each of the potential stellar complexes. This revealed that most of these large complexes are composed of well-defined smaller subgroups having sizes corresponding to typical OB associations. This suggests a hierarchical arrangement of OB stars groupings, already found previously in other galaxies (Efremov et al. 1987; Battinelli et al. 1996).

Once we will have an accurate distance determination to NGC 300 from our project, we will use the results of this paper to compare the system of $\mathrm{OB}$ associations of NGC 300 to those of other galaxies for which such studies have been performed.

Acknowledgements. We would like to thank the OGLE team, especially Drs.: Andrzej Udalski, Michal Szymański and Marcin Kubiak for their kind help in observations and data reduction. We are very grateful to the European Southern Observatory for large amounts of observing time with the Wide Field Camera on the $2.2 \mathrm{~m}$ telescope. It is a pleasure to thank the $2 \mathrm{p} 2$ team for expert support and for doing several of the observations in service mode. It is also a pleasure to express our gratitude to Dr. Paolo Battinelli for providing us with his computer programs. WG acknowledges financial support through Fondecyt grant No. 1000330. We would also like to thank Dr. Azzopardi for valuable comments. 


\section{Appendix A: Selected maps of associations}
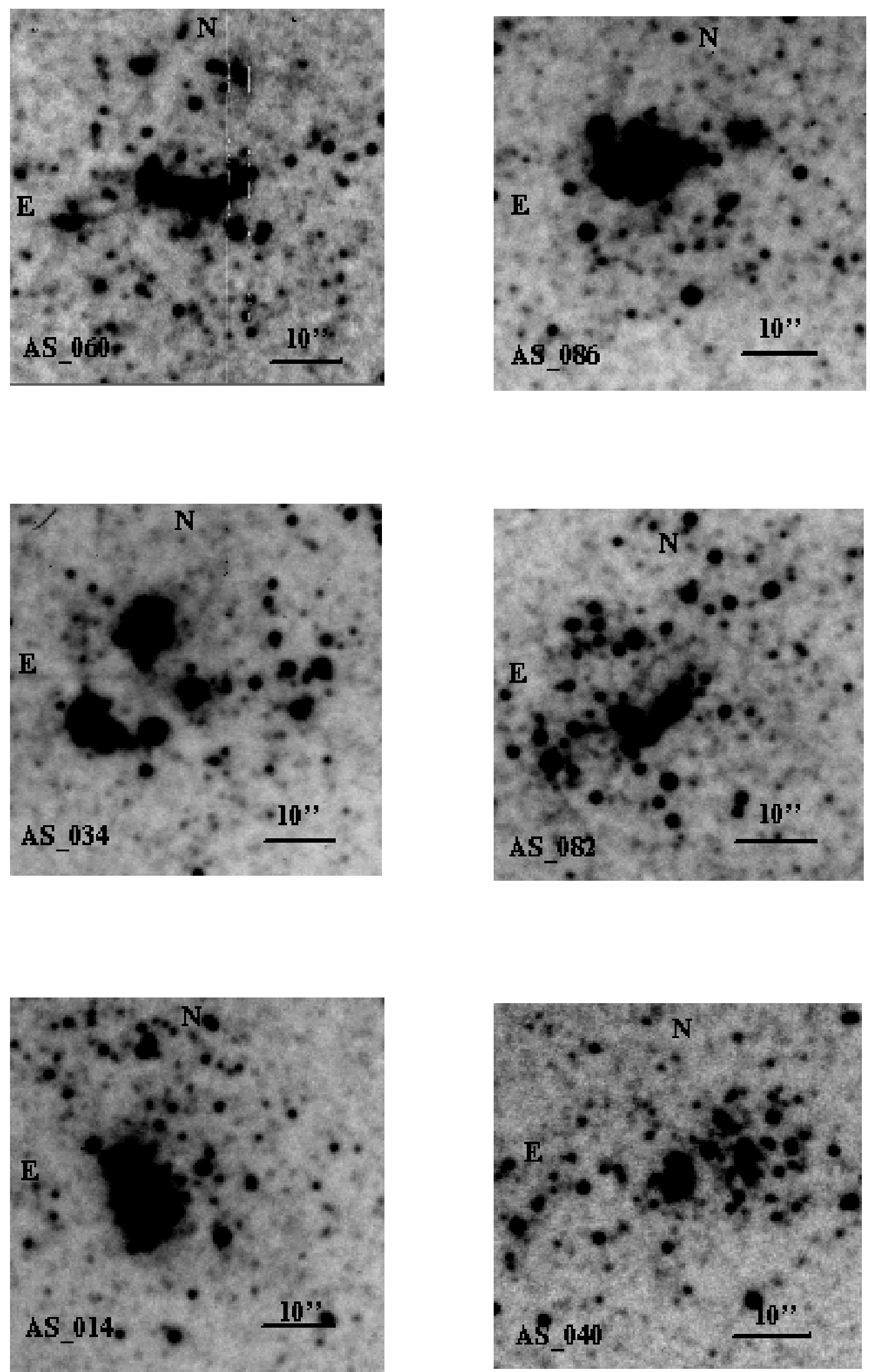

Fig. A.1. Maps of 6 typical associations in NGC 300 


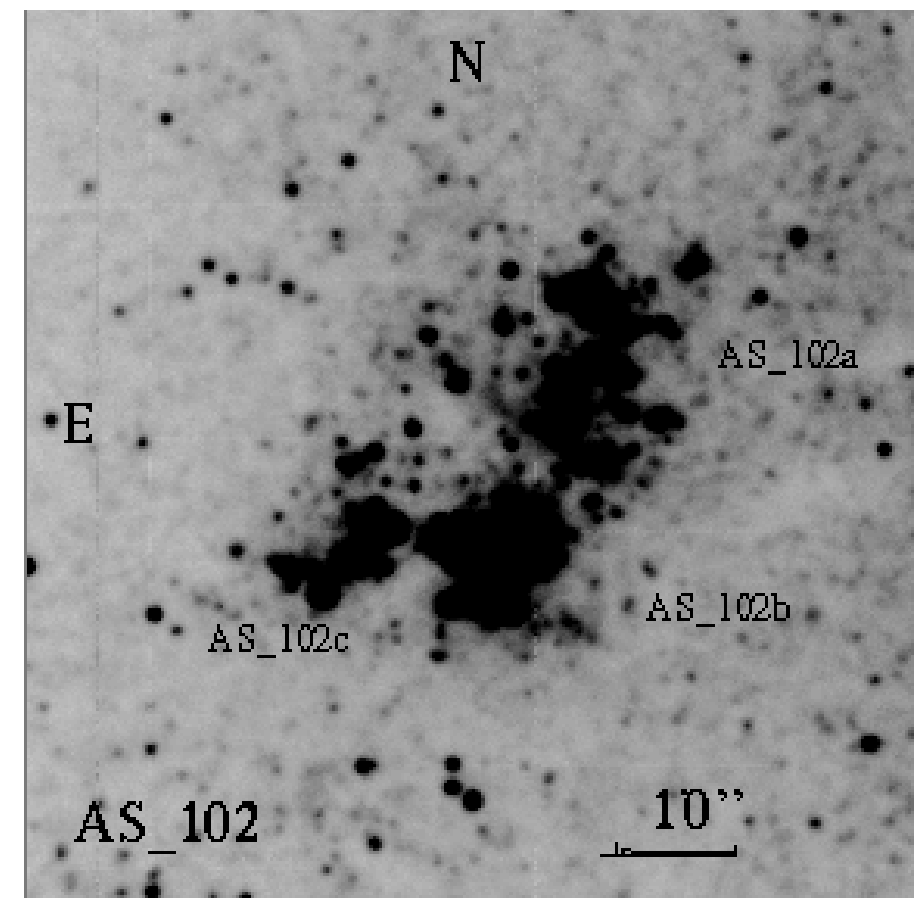

Fig. A.2. Map of one potential stellar complex in NGC 300

\section{References}

Battinelli, P. 1991, A\&A, 244, 69

Battinelli, P., Efremov, Y., \& Magnier, A. E. 1996, A\&A, 314, 51

Breysacher, J., Azzopardi, M., Testor, G., \& Muratorio, G. 1997, A\&A, 326, 976

Deharveng, L., Caplan, J., Lequeux, J., et al. 1988, A\&AS, 73, 407

Efremov, Y. N., Ivanov, G. R., \& Nokolov, N. S. 1987, Ap\&SS, 135,119

Freedman, W. L., Madore, B. F., Hawley, S. L., et al. 1992,
ApJ, 396, 80

Hodge, P. W. 1986, in Luminous Stars and Associations in Galaxies, IAU Symp. 116, ed. C. W. H. De Loore, A. J. Willis, \& P. G. Laskarides (Reidel; Dordrecht), 369 Landolt, A. U. 1992, AJ, 104, 372

Magnier, E. A., Battinelli, P., Lewin, W. H. G., et al. 1993, A\&A, 278, 36

Udalski, A., Kubiak, M., \& Szymański, M. 1997, Acta Astron., 47, 319

van den Bergh, S. 1992, PASP, 104, 861

Wilson, C. D. 1991, AJ, 101, 1663

Wilson, C. D. 1992, ApJ, 386, L29 